\title{
Distortion of a Toroidal Field by Convection
}

\author{
D. W. Allan and E. C. Bullard \\ Cambridge University, Cambridge, England
}

$I^{N}$ $\mathrm{N}$ a perfectly conducting body the lines of magnetic force move with the material; the total number crossing the surface in either direction is therefore constant, and cannot be changed by motions of the material forming the body. Both in terrestrial magnetism and in sunspots, large changes in the external field are observed which do involve a change in the total number of lines and cannot be explained by a mere crowding together of lines of force that already cross the surface. In these phenomena loops of field must emerge through the surface, and the theory must involve the finite conductivity of the material.

A theory of the secular variation of the earth's magnetic field in which the changes of field are caused by electromagnetic induction in the moving material of the core is in many ways attractive, particularly as it gives some hope of explaining the short time scale and complex pattern of the changes. In detail the theory has been less successful. Bullard ${ }^{1}$ has considered induction in eddies idealized as rotating spheres. If the eddy rotates in a field equal to the dipole field (about 4 gauss at the surface of the core) the calculated effect is too small no matter how fast the eddy rotates. The dynamo theory provides a stronger field in the core, perhaps as much as several hundred gauss. This field, however, is "toroidal" and vanishes at the surface of the core; the eddy must therefore be at some depth if this toroidal field is to be the source of the induction and the time for the field to grow will be greatly lengthened.

We have recently investigated the possibility of the strong toroidal field being brought to the surface by a rising convection current. By this means the strong field can be brought from a depth $d$ in a time $d / v$, where $v$ is the vertical velocity. Without this means of transport the field would take a time of order $4 \pi \kappa d^{2}$ to diffuse from a depth $d$ through material of conductivity $\kappa$.

${ }^{1}$ E. C. Bullard, Monthly Notices Roy. Astron. Soc. Geophys. Suppl. 5, 248-257 (1948).
If $v$ is $0.1 \mathrm{~cm} / \mathrm{sec}, d$ is $300 \mathrm{~km}$, and $\kappa=3 \times 10^{-6}$, the times are 10 and $1000 \mathrm{yr}$.

Hydromagnetic theory is considerably complicated if it is necessary to take account of finite conductivity. In the present problem, however, it may be a fair approximation to regard the disturbance as small compared to the initial field. This simplification makes it possible to discuss a number of simple cases analytically. The easiest is the effect of a specified convection current in two dimensions distorting a horizontal magnetic field which is constant in any horizontal plane. This problem has been worked out in detail. The time to reach final equilibrium is very long indeed. This unexpected result appears to be due to the initial loop of field being pushed through the surface fairly quickly and then very slowly widening by lateral diffusion. It seems likely that in the earth's core this very slow process never has time to come to completion and that the convection currents change their form or move before more than the initial stages are complete. The magnitude of the field, even in the initial quickly growing phase, is quite adequate to explain the secular variation if the velocities in the core are of the order of a millimeter per second. We hope to investigate the effect of the approximations made in the analytical solution by a numerical solution of the original equations in some special case.

If the inertial forces are neglected, the temperature field necessary to maintain the convective motion against the electromagnetic forces can be calculated; it involves temperature differences of the order of $10^{-3}{ }^{\circ} \mathrm{C}$ which raises no special difficulty.

The distortion of a magnetic field by a convective motion appears to provide a number of soluble problems of possible astronomical and geophysical interest. In addition to the two-dimensional problem described above we have solutions for a convection current with cylindrical symmetry and specified velocity distribution and for convection in a sphere.

\section{DISCUSSION}

E. N. PARKER, Enrico Fermi Institute for Nuclear Studies, University of Chicago, Chicago, Illinois: I want to take exception to the statement that given a perfectly conducting fluid body containing an internal magnetic field, it is not possible to get any of that field outside of the body. This is true if you have a rigid boundary such as the core of the earth, but not for a free boundary. Suppose we consider the surface of a star with the lines of the force running horizontally underneath the surface. If one wishes to extract these lines of force, he postulates some convective motion which conveys the lines of force in a rising surge of fluid above the surface of the star. Then, since there is no Lorentz force along the lines of force, the fluid, under the effects of gravity, simply drains out of the raised region along the lines of force, leaving the field behind. 
E. C. BUllaRD, Cambridge University, Cambridge, England: Yes, I think this has a very great relevance to the sunspot problem, with the interesting question being how much of the magnetic energy this process gets rid of. The sun is a very large body and the time constant, if you take the square of the radius and multiply it by the conductivity, is of the order of $10^{10} \mathrm{yr}$. If there is no other process for getting rid of the field, it will last for $10^{10} \mathrm{yr}$. This process of blowing the magnetic field away obviously gets rid of a certain amount of magnetic field by blowing it into regions where the conductivity is low and it can decay. However, it is not clear whether this process decreases or increases the total magnetic energy within the body. It probably increases it, because in forming a loop of field the lines of force are stretched.

L. BIERMANN, Max Planck Institut für Physik, Göttingen, Germany: I just want to comment on the statement that has been made with respect to sunspots. The conductivity in the sunspots is high, even taking into regard the low degree of ionization and the possibility of ambipolar diffusion. For the present purposethat is the appearance or disappearance of sunspots-it is so high that practically one can say that the magnetic flux through a given mass is constant, and therefore the spots appear by mass motions. This explanation is effectively equivalent to the one given by Cowling many years ago.

E. C. BullaRD : Yes, most of the lines of force must disappear into the sun, but surely if you look at the prominences it does look as if some of it goes upward.
A. SCHLÜTER, Max Planck Institut für Physik, Göttingen, Germany: But the material in this "upward" region has even better conductivity than the photosphere.

W. D. HAYES, Department of Aeronautics, Princeton University, Princeton, New Jersey: With respect to this, if the material that is blown up goes back into the surface and the highly conductive stellar atmosphere with which the material is replaced were to carry no magnetic field, the atmosphere would automatically carry a region of no magnetic field back into the region of interest. So it would be impossible to leave the lines of force sticking up above the surface as long as this atmosphere is of essentially infinite conductivity.

G. K. BATCheloR, Trinity College, Cambridge, England: There is one comment that I expect some of the fluid mechanics people have at the back of their minds about this work that has been described; viz., in the immediate neighborhood of the stagnation point an exact solution is possible. This may not be widely known to the astrophysicists, although it is a fairly simple and easily worked out. solution. Very near the stagnation point (and I am assuming now that the boundary is not deformed very much by the uprising current), the velocity has a universal form, the two components of the velocity being linear in the components of distance from the stagnation point. In this same region, the lines of magnetic force are straight and parallel to the boundary, and the action of the fluid motion there is a simple stretching one. The convective increase in the magnetic field there is balanced exactly by the decrease due to electric conduction. In that neighborhood, one can write down the exact form of $\mathrm{H}$ without difficulty. 